\title{
CIDADES RESILIENTES: UM MODELO DE AÇÕES INOVADORAS VOLTADO À EVENTOS HIDROLÓGICOS
}

\section{ESILIENT CITIES: A MODEL OF INNOVATIVE ACTIONS AIMED AT HYDROLOGICAL EVENTS}

\author{
Thuany Nascimento Domingues, Daiane Maria De Genaro Chiroli
}

Universidade Tecnológica Federal do Paraná

\section{RESUMO}

Os desastres hidrológicos movidos por eventos climáticos têm se tornado mais frequentes, sendo obstáculos complexos para o desenvolvimento de ferramentas e planos de ações mais efetivos nas cidades paranaenses. Assim, para promover melhorias ao sistema de gestão, esse trabalho tem por objetivo principal fortalecer as políticas de resiliência propondo ações inovadoras para superar os riscos e vulnerabilidades no enfrentamento à eventos hidrológicos futuros. Para isso, foi realizada uma revisão sistemática da literatura por meio do Methodi Ordinatio e por um modelo construído a partir da metodologia DMAIC. Na revisão sistemática, encontrou-se lacunas de conhecimento voltados ao tema em questão, e o uso da metodologia DMAIC se mostrou satisfatório para compilar as informações levantadas e também, para a construção do modelo, buscando colaborar para uma visão clara e estratégica a longo prazo no contexto de resiliência nas cidades paranaenses.

\section{Palavras Chave}

Inovação; Cidades Resilientes; Eventos Hidrológicos; DMAIC

\begin{abstract}
Hydrological disasters driven by climatic events have become more frequent, being complex obstacles to the development of more effective tools and action plans in the cities of Paraná. Thus, to promote improvements to the management system, this work has as main objective to strengthen resilience policies by proposing innovative actions to overcome risks and vulnerabilities in coping with future hydrological events. For this, a systematic review of the literature was performed through Methodi Ordinatio and by a model constructed from the DMAIC methodology. In the systematic review, knowledge gaps focused on the theme in question were found, and the use of the DMAIC methodology proved satisfactory to compile the information raised and also to build the model, seeking to collaborate for a clear and strategic long-term vision in the context of resilience in the cities of Paraná.
\end{abstract}

\section{KEY WORDS}

Innovation; Resilient Cities; Hydrological Events; DMAIC 


\section{INTRODUÇÃO}

Os desastres naturais resultantes por eventos climáticos têm se tornado mais frequentes em todo o mundo, sendo obstáculos complexos para o desenvolvimento urbano das cidades (Folke, 2002). Entre 2000 e 2019, foram registrados pelo Emergency Events Database (EM-DAT), 7.348 eventos de desastres que afetaram mais de 4 bilhões de pessoas, promovendo U\$2,97 trilhões em perdas para a economia global (UNISDR, 2009).

No Brasil, o Centro Universitário de Estudos e Pesquisas sobre Desastres da Universidade Federal de Santa Catarina (CEPED/UFSC) registrou, de 1995 a 2014, um prejuízo de 182 bilhões de reais. Somente no Estado do Paraná, foram 4.630 registros de desastres que afetaram 8 milhões de pessoas nos 380 municípios, ocasionando um custo de 6,8 bilhões de reais aos cofres públicos, no período de 2010 a 2018 (Asad et al., 2021). Segundo esses dados, 40\% desses desastres são caracterizados por enchentes, alagamentos e inundações. Em todo o mundo, essa categoria é considerada como um dos perigos naturais mais persistentes que ameaçam o design e planejamento urbano nas cidades (Young, 2014). Além disso, os desastres hidrológicos são mais facilmente previstos e prevenidos que outros, tornando-os adequados para o desenvolvimento de ferramentas e planos de ações mais efetivos (Rodríguez-Espíndola et al., 2018). Assim, as governanças e administrações públicas vêm incorporando medidas e estratégias para a Redução de Risco de Desastres (RRD) nas cidades (DíazDelgado; Iniestra, 2014).

Para maximizar a redução desses efeitos, o fortalecimento de ações inovadoras e planejamentos para superar os riscos e vulnerabilidades, podem ser a melhor resposta para auxiliar as cidades em sua capacidade de resiliência frente à eventos futuros (Miao; Popp, 2014).

Atualmente, o conceito de resiliência vem sendo cada vez mais admitido e bastante utilizado no contexto de RRD, pois corresponde a uma rede sustentável de sistemas físicos e comunidades humanas, capaz de manter ou retornar rapidamente a seu funcionamento e desenvolvimento básico desejado diante de uma perturbação (Meerow et al., 2015). Uma cidade resiliente tem a responsabilidade de adotar, a longo prazo, medidas que propiciem a capacidade adaptativa de preparação, aprendizagem e auto-organização, a fim de reduzir as vulnerabilidades - social, ambiental e econômica - existentes na ocorrência de desastres (Santos, 2009). Para isso, iniciativas internacionais, como a Campanha Construindo Cidades Resilientes (CCCR) do Escritório das Nações Unidas para a RRD, lançada em 2010, vem assumindo uma posição de destaque, já que define, 
claramente, estratégias e diretrizes em nível local, para promover a resiliência a desastres a partir de dez passos essenciais (UNISDR, 2012).

No sentido de buscar iniciativas para desenvolver a resiliência a desastres nas cidades, a metodologia Definir, Medir, Analisar, Melhorar e Controlar (DMAIC - Define, Measure, Analyse, Improve, Control) é uma ferramenta facilitadora na obtenção de melhoria inovadora. Essa metodologia surgiu com o advento do Six Sigma na década de 1980, um sistema de gestão que visa reduzir a variabilidade de processo, e é reconhecida por grandes e pequenas instituições como a mais eficaz em buscar sistematicamente a melhoria contínua de processos, trazendo benefícios significativos para organizações com sua implementação (Treichler, 2005). É baseada em um ciclo de melhorias que se inicia com a definição de um problema (Fase Define), seguido pela medição do desempenho da linha de base, com ênfase em características relacionadas ao potencial de resiliência das cidades (Fase Measure), e então, variáveis-alvo são identificadas e mapeadas contra os preditores potenciais por meio de ferramentas apropriadas para determinar configurações do processo (Fase Analyse) e para, finalmente ser possível buscar melhorias no desempenho do processo (Fase Improve) a partir da sustentação e imposição de planos de controle eficazes (Fase Control) (Sokovic et al., 2010).

Sendo assim, o presente estudo possui dois objetivos. Em primeiro lugar, realizar uma revisão sistemática da literatura a fim de entender e levantar as questões e aspectos mais abordados no contexto de gestão de desastres hidrológicos, cidades resilientes e inovação, com o intuito de analisar a abordagem utilizada pelo Estado do Paraná. E segundo, propor um modelo de ações inovadoras que contribua para as cidades paranaenses se tornarem mais resilientes frente a eventos hidrológicos.

\section{MATERIAIS E MÉTODOS}

A fim de desenvolver a estrutura e atender os objetivos dessa pesquisa, organizou-se o trabalho em duas vertentes: revisão sistemática da literatura e desenvolvimento de um framework de ações inovadoras voltado à eventos hidrológicos para cidades resilientes.

\subsection{Revisão Sistemática da Literatura}

Para realizar a revisão sistemática da literatura, foram analisados artigos completos com base em critérios pré-estabelecidos utilizando a metodologia Methodi Ordinatio, conforme Figura 1. 


\section{¿UISUI}

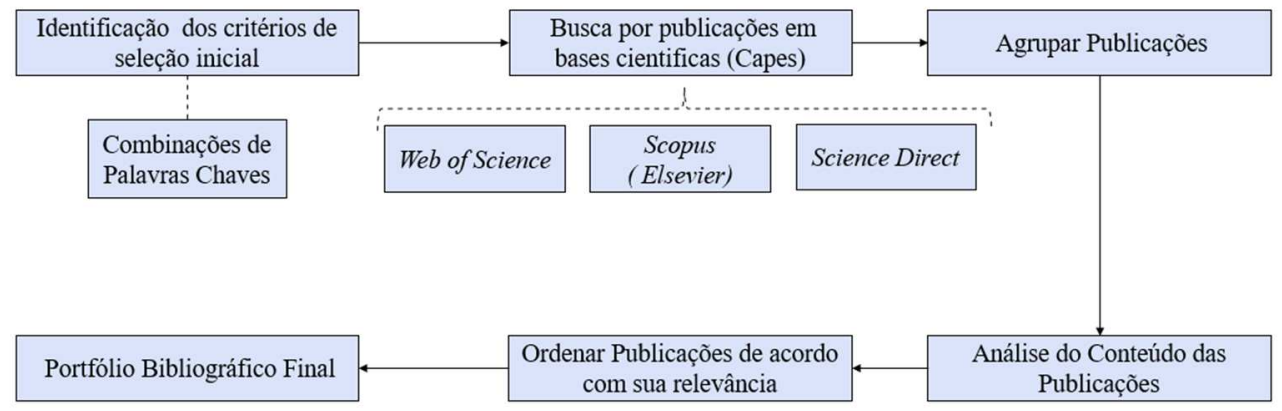

Figura 1 - Fluxo sistemático para a pesquisa bibliográfica. Fonte: os autores (2021)

A triagem das bases de dados foi realizada no ambiente da plataforma da Comissão de Aperfeiçoamento de Pessoal do Nível Superior (CAPES) por meio da permissão de acesso com a licença acadêmica. Assim, foram selecionadas três bases de busca: Web of Science, Scopus (Elsevier) e Science Direct.

Nessas bases, conforme trabalho de Barros (2020), foram realizadas buscas por palavras-chaves que se relacionavam com o tema. Após a seleção destas palavras, realizou-se a combinação entre elas de forma a atender os três focos principais deste trabalho: gestão de desastres hidrológicos, cidades resilientes e inovação. Na Tabela 1 se apresentam os termos utilizados de busca construída para essa pesquisa.

Tabela 1: Palavras-Chaves da Pesquisa. Fonte: os autores (2021)

\begin{tabular}{|c|c|}
\hline Tema & Sequência de busca \\
\hline $\begin{array}{l}\text { Gestão de Desastres } \\
\text { Hidrológicos }\end{array}$ & $\begin{array}{l}\text { "Disaster Risk Management”, “Disaster Relief”, ”Disaster Risk } \\
\text { Assessment", "Disasters", "Disaster Risk Reduction”, "Flood risk } \\
\text { assessment", "Humanitarian Logistics" }\end{array}$ \\
\hline Cidades Resilientes & $\begin{array}{l}\text { "Resilient Cities", “Urban Resilience", "Resilience”, "Community } \\
\text { Resilience". }\end{array}$ \\
\hline Inovaç & “Innovation Management”, “Innovation”. \\
\hline Outros Relacionados & $\begin{array}{l}\text { "Framework", "Municipal Management", "Flood resilience", "Sendai } \\
\text { Framework", "DMAIC", "Six Sigma", "Smart Cities". }\end{array}$ \\
\hline
\end{tabular}

A realização das combinações foi feita utilizando aspas duplas para a busca da palavra exata, bem como o uso de operadores boleanos de lógica e proximidade AND/OR e NOT, para a inserção e negativação, respectivamente, das palavras-chaves. Na busca por publicações, foi considerada uma 
faixa de 10 anos compreendendo os anos de 2010 a 2021. A seleção dos artigos de maior impacto e autores relevantes foi realizada por meio da ferramenta Bibliometrix e pelo software gratuito RStudio. Os artigos encontrados foram exportados das bases de dados como arquivo BibTex e analisadas por meio do Methodi Ordinatio que permite uma revisão sistemática de literatura baseada nos fatores: ano de publicação, fator de impacto e número de citações. Por fim, foram lidos todos os títulos e resumos dos artigos do portfólio bibliográfico final, e a partir de então foram desenvolvidos os resultados da pesquisa.

\subsection{Desenvolvimento do framework de ações inovadoras voltado à eventos hidrológicos}

Para a coleta de informações foram utilizados dados secundários (análise de documentos e modelos já existentes). A coleta dos dados secundários foi realizada por meio de (i) relatórios disponíveis das principais instituições envolvidas com o tema (CEMADEN e CENACID); (ii) sites e plataformas digitais públicos dessas instituições; (iii) artigos acadêmicos e; (iv) publicações em revistas especializadas.

$\mathrm{Na}$ análise dos dados, buscou-se descrever qualitativamente os pontos principais que relatam a realidade do sistema de gestão de desastres e cidades resilientes, utilizando como meio de tomada de decisão a metodologia DMAIC.

\section{RESULTADOS E DISCUSSÕES}

\subsection{Revisão Sistemática da Literatura}

A revisão intensiva da literatura sobre os campos relacionados, auxiliam a desenvolver um conhecimento para este trabalho de pesquisa. Assim, a análise dos conteúdos se deu, primeiramente, a partir de seus principais aspectos em termos do ponto de vista acadêmico e social. No eixo acadêmico, observou-se a abertura de janelas de oportunidades em ações inovadoras voltadas para a gestão de RRD e cidades resilientes, uma vez que se evidenciou uma lacuna de trabalhos relacionados a temática deste estudo.

A fim de priorizar as buscas, devido as inúmeras possiblidades de combinações, foi considerado como foco principal a combinação das palavras-chaves: "Urban Resilience", "humanitarian logistics", innovation". No recorte temporal dos últimos 10 anos, nas bases internacionais Web of Science, Scopus e Science Direct, foram retornados 46 trabalhos relacionados a cidades resilientes, 
174 a logística humanitária e 1.044 a inovação, todas sob o contexto de gestão de desastres. No entanto, quando esses temas foram buscados em conjunto, não houve o retorno de nenhuma pesquisa.

Essa lacuna bloqueia o desenvolvimento do conhecimento científico, o qual poderia auxiliar as administrações públicas na criação de planos de melhoria mais adequados e eficazes para lidarem com os desastres hidrológicos. A fim de contribuir para a análise, foram selecionados outros trabalhos, por relevância e objetivos, que se alinhassem a esta pesquisa. Os principais autores dos artigos analisados foram categorizados por tema estudado (Tabela 2).

Tabela 2: Autores dos principais artigos coletados Fonte: os autores (2021)

\begin{tabular}{cl}
\hline Temas & \multicolumn{1}{c}{ Autores } \\
\hline Gestão de Desastres & (Asad et al., 2021); (Young, 2014); (Rodríguez-Espíndola et al., \\
Hidrológicos & $\begin{array}{l}\text { 2018); (Díaz-Delgado; Iniestra, 2014); (Alem et al.,2016); (Izumi } \\
\text { et al., 2019); (Jameson; Baud, 2016) }\end{array}$ \\
& (Folke, 2002); (Meerow et al., 2015); (Santos, 2009); (UNISDR, \\
2012); (UNISDR, 2015); (Lima, 2020); (Hofmann, 2021); (Liao \\
et al., 2016) \\
(Miao; Popp, 2014); (Hu et al., 2018); (Ahern et al., 2014); (Gault, \\
2018); (Kahn, 2018) \\
Inovação & (Werkema, 2012); (Sokovic et al., 2010); (Neubauer et al., 2019); \\
& (Ghosh; Maiti, 2012); (Spirn, 2014); (Desouza; Flanery, 2013) \\
\hline
\end{tabular}

Já no contexto social, é importante considerar que a eventualidade dos fenômenos hidrológicos, bem como seus desdobramentos, o que fortalece a necessidade de estudos que auxiliem nas formas de enfrentá-los com o mínimo de perdas humanas e econômicas, garantindo assim, a redução das vulnerabilidades das populações e a volta das cidades ao seu funcionamento (Ahern et al., 2014).

Alguns pontos importantes acerca da temática foram identificados por meio da análise do conteúdo dos artigos selecionados na Tabela 2. Primeiramente, apenas os autores Díaz-Delgado e Iniestra (2014), Jameson e Baud (2016) e Liao et al. (2016) realizaram uma abordagem focada em desastres hidrológicos, mais especificamente em inundações. Pode-se destacar Liao et al. (2016) por trazer o tema no contexto de resiliência nas cidades. Estes autores defendem que, para promover a resiliência às inundações é necessário realizar medidas de prevenção de danos nas cidades, tendo como argumentos para seu modelo, três princípios focados no design urbano: (i) antecipar e acomodar as 
inundações; (ii) incorporar o processo ecológico das inundações; e (iii) relevar a dinâmica das inundações para o público.

Sob o ponto de vista da inovação enquadram-se os autores Hu et al. (2018), Miao e Popp (2014) e Ahern et al. (2014), sendo os dois primeiros em relação aos desastres e o último à resiliência. Hu et al. (2018) apresentam tentativas de investigar respostas inovadoras aos desastres climáticos ocorridos na China moderna no período de 2005 a 2013 e Miao e Popp (2014) estudaram como as inovações respondem a choques de desastres naturais. Esses estudos afirmam que a ocorrência dos desastres climáticos estimula a inovação nos campos de mitigação de desastres, mas não em outros, o que infere a necessidade das políticas públicas e governos incentivarem e aumentarem o investimento nos setores de pesquisa e desenvolvimento tecnológico para antes e após desastres climáticos. Já Ahern et al. (2014), retrataram que apesar de haver medidas e diretrizes enfatizando a resiliência, elas não motivam ou apoiam as inovações necessárias para auxiliar o desenvolvimento urbano e a melhorar a infraestrutura dos municípios.

Com base nos estudos citados, tem-se que as inovações impulsionam o crescimento e ajudam a enfrentar os desafios impostos pela ocorrência de desastres e a trazer a resiliência às cidades. As inovações podem assumir formas diferentes e não precisam ser inteiramente novas ou radicais por natureza, podendo surgir como produtos, processos, conceitos, métodos, abordagens e outros tipos, possuindo, nesse contexto, objetivos não apenas econômicos, mas capazes de salvar vidas, reduzir perdas e promover a coesão social e o desenvolvimento sustentável.

Quando entramos no contexto de gestão pública e metodologias de melhoria, apenas Neubauer et al. (2019) abordou o tema. Essa pesquisa apresentou um estudo qualitativo sobre gestão tecnológica, utilizando como base a metodologia DMAIC. O autor ressalta que essa abordagem é apropriada para propor práticas inovadoras na melhoria do sistema de gestão. Além de frisar a importância de elevar o conhecimento das partes envolvidas, desenvolvendo suas competências e habilidades.

Diante das informações levantadas, observa-se que apesar dos trabalhos citados não apresentarem conexão direta com os temas principais, eles foram extremamente importantes para exemplificar e fomentar a necessidade do conhecimento, do envolvimento e do investimento das instituições públicas em ações inovadoras voltados para a gestão de desastres e a busca por resiliência. Porém, fica evidente a escassez de informações em relação à integração dos temas abordados no recorte temporal estudado. Tendo em vista a importância da criação de medidas mais assertivas na gestão de 
RRD, LH (Logística Humanitária), cidades resilientes e inovação, mais pesquisas nesses contextos são fundamentais.

\subsection{Desenvolvimento do framework de ações inovadoras voltado à eventos hidrológicos}

A coleta dos dados secundários foi dividida em quatro partes principais: (i) estudo de caso de desastres hidrológicos no Estado do Paraná; (ii) conceito de cidades resilientes, (iii) evolução do conceito de cidades resilientes no Estado do Paraná; e (iv) construção da proposta desenvolvida a partir da metodologia DMAIC.

\subsubsection{Estudo de caso de desastres hidrológicos no Estado do Paraná}

A gestão de riscos de desastres no Estado do Paraná conta com o Sistema Informatizado de Proteção e Defesa Civil (SISDC), uma ferramenta desenvolvida pela Coordenadoria Estadual de Proteção e Defesa Civil (CEPDEC) em conjunto com a Companhia de Tecnologia da Informação e Comunicação do Paraná (CELEPAR) (CEPED, 2012).

O mapeamento das áreas de atenção foi iniciado em 2012 e até o momento o Estado conta com 285 mil residências e aproximadamente 1,2 milhão de pessoas expostas a alagamentos e inundações. Essas áreas foram ganhando relevância para os gestores de proteção e defesa civil no Paraná com o decorrer dos anos, uma vez que no início os esforços eram voltados para o mapeamento dessas áreas (até 2014) para em seguida qualificar os dados e informações destes mapeamentos (de 2014 em diante). Em relação à quantidade de desastres ano a ano, no período de 2012 a 2018 foram registrados 3.764 desastres, que custaram mais de R \$ 5,9 bilhões ao Estado para reestruturação das cidades.

Esses números demonstram uma urgência de que o planejamento dos municípios seja fundamentado na identificação dos riscos de desastres e legitimados pelas instituições governamentais e população, a fim de reduzir os efeitos atrelados à crescente urbanização e desencadear a auto-organização, aprendizagem e adaptação (Walker; Salt, 2012). Para isso, é necessário que o mapeamento e a identificação dos riscos forneçam informações confiáveis e oportunas, de forma prática e acessível, com vistas a tornar a gestão de risco mais inclusiva à população, potencializando a construção de municípios mais resilientes.

O Estado do Paraná possui 20 municípios com o maior índice de risco a desastres hidrológicos (Figura 2). O índice de risco foi calculado por meio da multiplicação da periculosidade e 


\section{GESTÃO \& SUSTENTABILIDADE AMBIENTAL

vulnerabilidade, obtendo-se uma escala de 1 a 25 dividida em 5 classes para municípios com: risco baixíssimo (1- 5), risco baixo $(6-10)$, risco médio $(11-15)$, risco alto $(16-20)$ e risco altíssimo $(21-25)$.

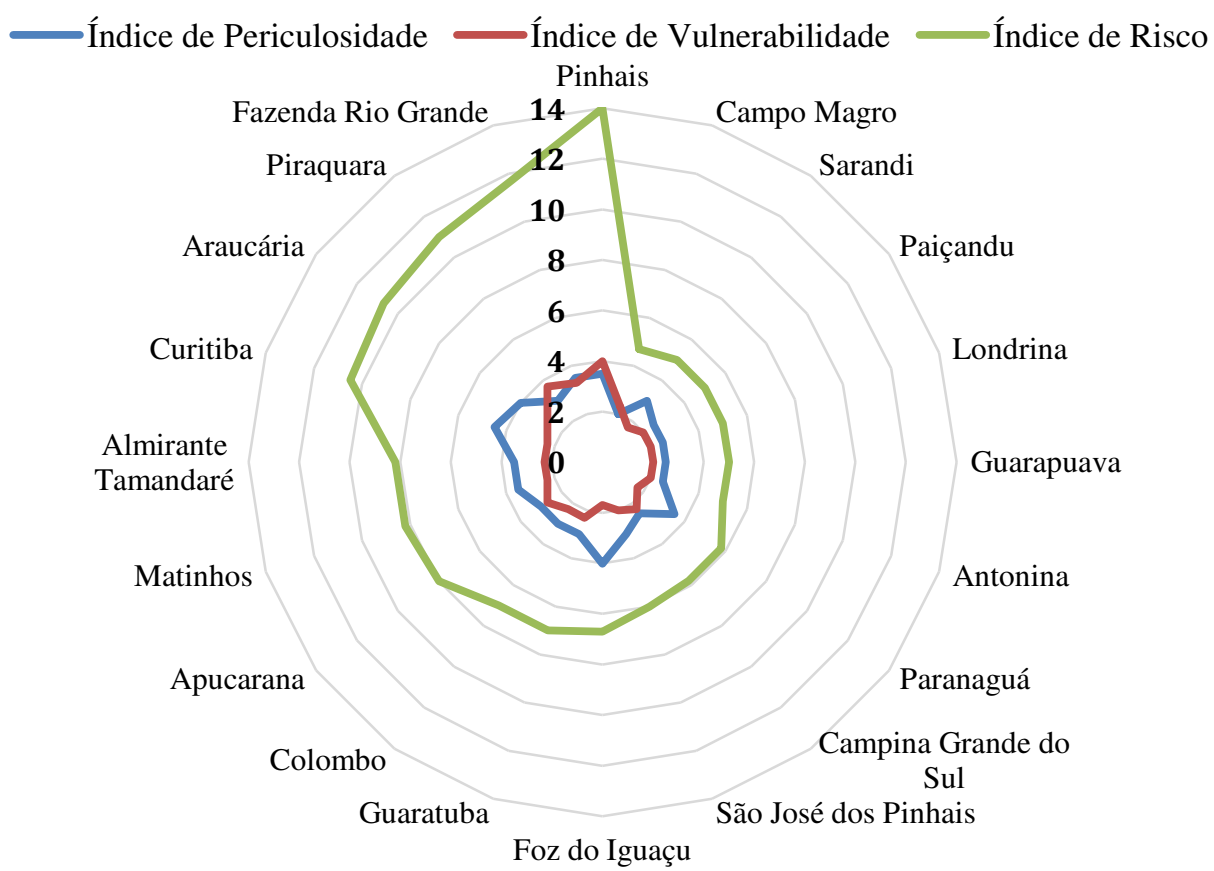

Figura 2 - Índices relacionados à ocorrência de desastres hidrológicos dos 20 municípios do Estado do Paraná com maior risco. Fonte: Adaptado de Gonçalves e Fernandez (2016).

Observa-se que a maioria dos municípios do Estado possuem uma suscetibilidade muito baixa a esses tipos de desastres, podendo-se inferir que o Paraná não é um Estado com grandes riscos de ocorrência desses tipos de desastres. Todavia, tem-se uma crescente evolução do número de ocorrências e áreas de atenção, o que somado à população exposta nos municípios a estes tipos de desastres, indicam a necessidade de serem feitos planos de contingências para que tais eventos não venham a se tornar catástrofes (Kobiyama et al., 2006).

\subsubsection{Conceito de cidades resilientes}

O termo resiliência teve origem com ênfase na física (1807), sendo propagado para outras áreas como psicologia (1966), ecologia (1970), organizacional (1998) (Béné et al., 2012). Dada a sua multidisciplinaridade, atualmente essa linha de pensamento vem sendo utilizada para adaptações às mudanças climáticas e RRD, cada vez mais voltada para área de planejamento das cidades. No 
entanto, segundo Meerow et al. (2015) os significados e definições da palavra resiliência não são muito concisos devido à literatura diversificada em torno do tema. Desse modo, a definição de resiliência associada às cidades avançou para instituições internacionais de aconselhamento político (Tabela 3), as quais se enquadram no conceito norteador do presente trabalho.

Tabela 3: Definições de Cidades Resilientes. Fonte: Meerow et al. (2015)

\begin{tabular}{|c|c|}
\hline Instituição & Definição \\
\hline $\begin{array}{c}\text { International Council for Local } \\
\text { Environmental Initiatives } \\
\text { (ICLEI) }\end{array}$ & $\begin{array}{l}\text { Uma cidade preparada para absorver e recuperar-se de qualquer choque } \\
\text { ou estresse, mantendo suas funções essenciais, estruturas e identidade, a } \\
\text { fim de prosperar diante das mudanças contínuas. A resiliência nas } \\
\text { cidades requer identificar e avaliar riscos, reduzir a vulnerabilidade e } \\
\text { aumentar a capacidade adaptativa e a preparação de emergência. }\end{array}$ \\
\hline $\begin{array}{c}\text { United Nations Office for } \\
\text { Disaster Risk Reduction } \\
\text { (UNISDR) }\end{array}$ & $\begin{array}{l}\text { Capacidade de um sistema, comunidade ou sociedade exposto a riscos } \\
\text { de: resistir, absorver, acomodar, adaptar-se, transformar e recuperar dos } \\
\text { efeitos de um perigo em tempo hábil e eficiente, por meio da preservação } \\
\text { e restauração de suas funções básicas por meio da gestão de riscos. }\end{array}$ \\
\hline Rockefeller Foundation & $\begin{array}{l}\text { A resiliência é a capacidade dos indivíduos, comunidades e sistemas de } \\
\text { sobreviver, adaptar-se e crescer diante do estresse e dos choques, e até } \\
\text { mesmo se transformar quando as condições o necessitam. }\end{array}$ \\
\hline 100 Resilient Cities & $\begin{array}{l}\text { A resiliência urbana é a capacidade de indivíduos, comunidades, } \\
\text { instituições, empresas e sistemas dentro de uma cidade para sobreviver, } \\
\text { adaptar e crescer, independentemente dos tipos de estresses crônicos e } \\
\text { choques agudos que experimentam. }\end{array}$ \\
\hline
\end{tabular}

Dentre as definições destacadas, observa-se a convergência de elementos muito importantes. Todos abordam a resiliência como uma capacidade, ou seja, uma característica que pode ser construída e adquirida por cidades, comunidades ou organizações. Sendo as cidades capazes de resistir, absorver, adaptar, transformar, mudar, recuperar e se preparar a certos eventos hidrológicos a que estão expostos, ou até mesmo a possibilidade de ocorrerem.

Nesse contexto a resiliência necessita ser articulada nas escalas globais, nacionais e locais, sob o conceito de governança de risco, a fim de manter as funções das cidades e buscar melhorias na resposta a choques futuros. A distinção dessas escalas é importante para os formuladores de políticas, pois dependendo de onde for enquadrada, irá refletir ações e debates específicos. No entanto, essas ações e debates não são mutuamente exclusivos, mas complementares, ou seja, ações de níveis locais devem ser complementadas por quadros da política nacional e assim por diante. Assim, analisar a resiliência requer uma abordagem holística e completa do sistema ao qual está sendo implementada. 


\subsubsection{Evolução do conceito de cidades resilientes no Estado do Paraná}

O conceito de resiliência nas cidades paranaenses surgiu com o aumento rápido das catástrofes em meios urbanos, resultando em perdas humanas e econômicas muito significativas (Lima, 2020). Dessa forma, inúmeros esforços dos governos locais, autoridades urbanas, departamentos centrais e regionais, setor privado, sociedade civil, instituições de pesquisas e de ensino superior são necessários para criar um ambiente urbano mais resiliente, que visa o enfrentamento dos riscos de desastres.

A promoção da CCCR: Minha Cidade está se preparando!", divulgada pelo Escritório das Nações Unidas para Redução do Risco de Desastres (UNISDR) no ano de 2010, foi um meio encontrado pelo Estado do Paraná para desenvolver a resiliência e o enfrentamentos às vulnerabilidades promovidas pelos desastres. Essa campanha visa estimular os gestores locais a investirem no aumento da resiliência por intermédio de metas e objetivos que busquem atender os dez passos essenciais do Marco de Sendai para a RRD 2015-2010, tendo como objetivo a construção de cidades resilientes.

O termo resiliência no Brasil passou a ser inserido na legislação brasileira em 2012, com a instituição da Política Nacional de Proteção e Defesa Civil (PNPDEC), que permitiu o início do pensamento e da construção de medidas referentes a GRD no país. Essa política trouxe como inovação a integração de diversas políticas setoriais, como a de ordenamento territorial, desenvolvimento urbano, recursos hídricos, geologia, infraestrutura, saúde, educação, tendo em vista o desenvolvimento sustentável (Brasil, 2012).

A conscientização quanto ao investimento de ações que visem a RRD tem aumentado. Dentre os 399 municípios paranaenses classificados como prioritários para o risco de desastres, 321 deles estão inscritos na CCCR, garantido ao Estado a segunda posição como estado com maior número de municípios participantes no país.

Uma outra ação criada com o objetivo de desenvolver capacidades juntos aos setores públicos foi a Redesastre em 2014. A Redesastre é uma rede formada por 23 instituições públicas e privadas que cooperam entre si a fim de buscar a RRD no Paraná.

Em 2015, o Estado sediou o primeiro curso ministrado pela UNISDR sobre a temática do Marco de Sendai, ocasião em que houve a proposta de adaptar e traduzir o material didático (tradução do guia e do Scorecard) para a realidade local. A partir desse ano, foram ministrados diversos cursos nos municípios a fim de desenvolver capacidades para tornar as cidades mais resilientes, tendo como 
objetivo auxiliar os gestores locais a implantar, na prática, os dez passos, a fim de tornar as cidades mais seguras frente aos desastres (CEPED, 2018), com base em UNISDR (2012). Os 10 passos essenciais da CCCR são: (1) Organização para resiliência frente aos desastres; (2) Identificar, compreender e utilizar os cenários de riscos atuais e futuros; (3) Fortalecer a capacidade financeira para a resiliência; (4) Alcançar o desenvolvimento urbano resiliente; (5) Proteger as zonas naturais de amortização para melhorar as funções protetoras dos ecossistemas; (6) Fortalecer a capacidade institucional para resiliência; (7) Compreender e fortalecer a capacidade social para a resiliência; (8) Aumentar a resiliência das infraestruturas; (9) Assegurar uma resposta adequada e efetiva frente aos desastres; e (10) Acelerar o processo de recuperação e reconstruir melhor.

Observa-se que os passos essenciais da campanha são amplos, permitindo o estabelecimento de metas que assegurem uma gestão de riscos de desastres multifacetada. $\mathrm{O}$ ordenamento desses passos pode ser dado em três momentos. O primeiro voltado para governança corporativa e da cidade (passos 1 a 3), o segundo para planejamento integrado (os passos 4 a 8) e o terceiro para o planejamento da resposta (passos 9 a 10). Esses momentos vão ao encontro dos três caminhos cumulativos para um estado resiliente, abordados nos estudos de Meerow et al. (2015) e Béné et al. (2012): persistência, transição e transformação. Estes trazem a forma de como as instituições responsáveis devem enfrentar a ocorrência de desastres a fim de buscar a resiliência nas cidades.

\subsubsection{Construção da proposta por meio da metodologia DMAIC}

A partir das informações levantadas na revisão da literatura, observou-se que as cidades expostas a mais riscos de desastres hidrológicos são as que também concentram mais recursos, oportunidades e capacidade de inovação para se tornarem mais resilientes. Isso traria benefícios, econômicos, sociais e ambientais para a sociedade, por meio de investimentos na prevenção, adaptação e recuperação de choques e estresses. Além disso, quanto mais os atores locais compreendem os riscos aos quais sua cidade está sujeita, mais aptos estarão para agir em relação a eles.

Dessa forma, construiu-se a proposta com intuito de auxiliar as cidades a desenvolver sua estratégia de resiliência local por meio da metodologia DMAIC. Para apresentar a construção da proposta, foi desenvolvida uma estrutura conceitual a fim de ilustrar a sequência da metodologia utilizada no desenvolvimento deste trabalho, conforme o estudo de Creswell (2010), representado na Figura 3. 


\section{¿UNISUI}

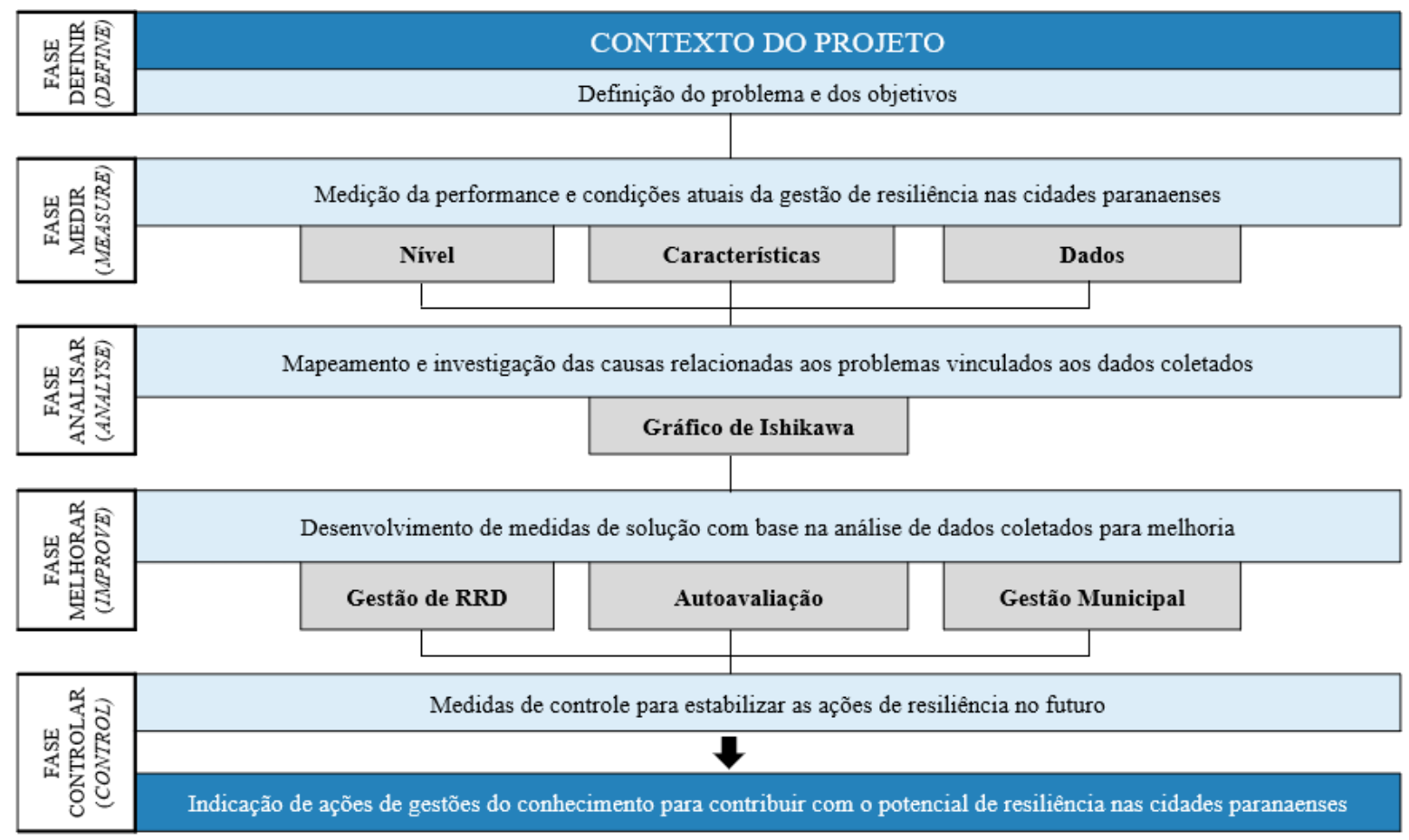

Figura 3 - Estrutura Conceitual da Proposta. Fonte: os autores (2021).

A definição do problema consistiu na observação da dificuldade das organizações paranaenses como a defesa civil, as prefeituras e até mesmo as instituições acadêmicas, entre outras, em melhorar a resiliência das cidades no estado frente aos riscos de alagamentos e inundações. De tal modo, para medir os níveis de resiliência e acompanhar o progresso das políticas voltadas a esse propósito, foram utilizados indicadores políticos, como índices de vulnerabilidade, áreas de riscos frentes a desastres hidrológicos, e utilizando o Scorecard, utilizou-se um conjunto de avaliações que permitem que os governos locais monitorem e analisem o progresso e os desafios na implementação do Marco de Sendai para a RRD, estruturado em torno dos objetivos da CCCR.

Embora o caminho para obtenção dessas ferramentas possa parecer comum, a tratativa e análise dos mesmos podem variar para cada cidade paranaense, uma vez que, cada cidade tem seus próprios objetivos e prioridades políticas, assim como riscos mais urgentes que irão influenciar no desenvolvimento de estratégias gerais. Atualmente, a metodologia utilizada pela Proteção e Defesa Civil do Estado do Paraná se baseia em cinco etapas: (i) Adesão à CCCR, (ii) Criação do comitê de resiliência Municipal, (iii) Criação do perfil Prevention Web, (iv) Autoavaliação pelo Scorecard de resiliência de desastres para as cidades e (v) Desenvolvimento do Plano Municipal de Resiliência. 
Mesmo com essa estrutura, há dificuldades em se implementar a resiliência nas cidades paranaenses. A fim de identificar as principais causas que influenciam esse objetivo, analisou-se o contexto atual por meio do mapeamento das ações com o diagrama de Ishikawa (Figura 4), conforme a fase Analisar da metodologia.

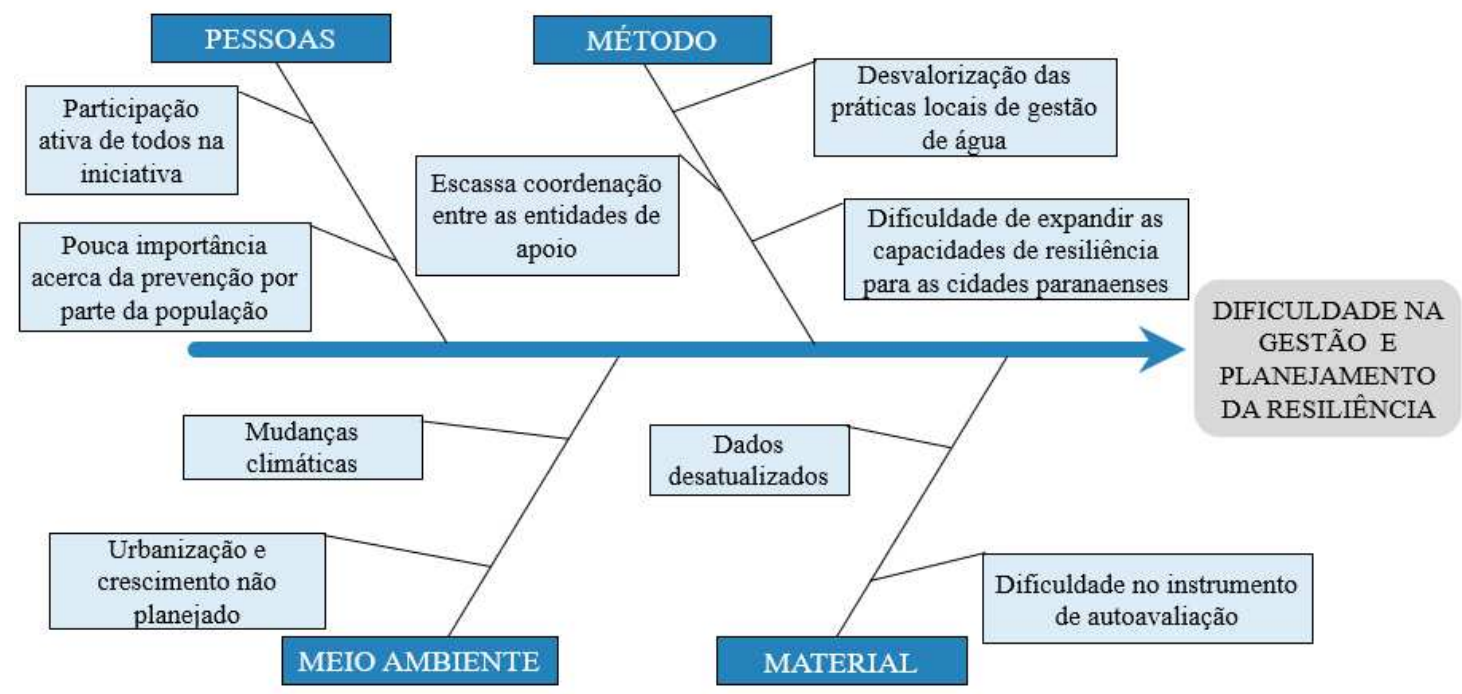

Figura 4 - Diagrama de Causa-Efeito. Fonte: os autores (2021)

Como resultado, observou-se claramente que existe um grande número de variáveis na gestão da resiliência, os quais impõem conflitos na performance dos objetivos. Relatos na literatura e as dificuldades encontradas por conta das lacunas de conhecimento acerca do assunto, auxiliaram na identificação dos parâmetros mais impactantes que causam as dificuldades na busca da resiliência frente aos desastres hidrológicos no diagrama de causa-efeito (Figura 4).

As inundações urbanas vêm crescendo devido a diversas problemas, como a proliferação de áreas urbanas sem desenvolvimento planejado, bem como às mudanças climáticas, além da desvalorização das práticas locais de gestão da água por parte dos municípios. E, apesar da existência de ferramentas como o Prevention web gerenciada pela UNISDR é importante atualizar e tornar disponíveis os dados locais e as tendências da RRD para que todas as partes interessadas possuam uma participação ativa nas iniciativas de resiliência nas cidades. Além do compartilhamento do instrumento Scorecard, que contém 117 indicadores com um score de 0 a 5, o que possibilita identificar os principais pontos positivos e negativos dos municípios. Essa dificuldade em expandir a importância e as capacidades de resiliência são destacadas dentre as 321 cidades participantes na CCCR no Estado do Paraná, sendo

\footnotetext{
Revista Gestão e Sustentabilidade Ambiental., v. 11, n. esp, p. 104-123, jan. 2022. (?) - II SIMPósIo 
que até o momento, apenas quatro se destacaram no processo de autoavaliação: Campo Largo, Curitiba, Primeiro de Maio e União da Vitória (UNISDR, 2017).

Com o levantamento das ações voltadas as fases Melhorar e Controlar, foi possível elaborar a proposta deste trabalho em um formato de um modelo (Figura 5) que contempla um objetivo principal, fases de aplicação e suas respectivas as etapas.

Sequencialmente, as fases sugeridas pelo framework abordam: (1) identificação, (2) caracterização, (3) análise e (4) identificação de estratégias. A primeira fase tem por objetivo levantar as características da cidade quanto aos riscos e os impactos dos desastres hidrológicos, para assim, definir preliminarmente uma estrutura estratégica. Isso se deve ao fato que, na análise dos estudos na área de resiliência aplicada no contexto das cidades, a identificação dos principais riscos frente a estes desastres, demostraram lacunas operacionais e de desenvolvimento, as quais precisam ser trabalhadas e incluídas na gestão local. Essa identificação é essencial para compreender o ambiente em que os recursos locais estão sendo incorporados dentro da organização.

A fase 2 consiste em mapear as ações inovadoras com base nos 4 pilares da resiliência, extraído das definições do termo no contexto deste trabalho. A visão de antecipar, responder, monitorar e aprender, possibilita uma abordagem prática e lógica no desenvolvimento do planejamento estratégico. O levantamento de medidas inovadoras já existentes serve como um meio de potencializar, não só a CCCR, mas também a parceria entre gestores públicos municipais, estaduais, regionais e também o setor privado, com o intuito de implementar a resiliência na cidade. Um exemplo de ação inovadora envolvendo essas partes interessadas é a criação de uma websérie que conta a história de cidades modelos enfatizando as características, possibilidades, competências, bem como as dificuldades na construção da resiliência. Essa ação utiliza a educação à distância $(\mathrm{EaD})$ para capacitar os tomadores de decisões nas cidades e reforçar os aspectos e esforços no desenvolvimento de uma cidade resiliente frente aos desastres hidrológicos. 


\section{ounisul}

\section{OBJETIVO}

Propor um modelo de açōes inovadoras voltado as cidades paranaenses a buscarem mais resiliência frente à eventos hidrológicos

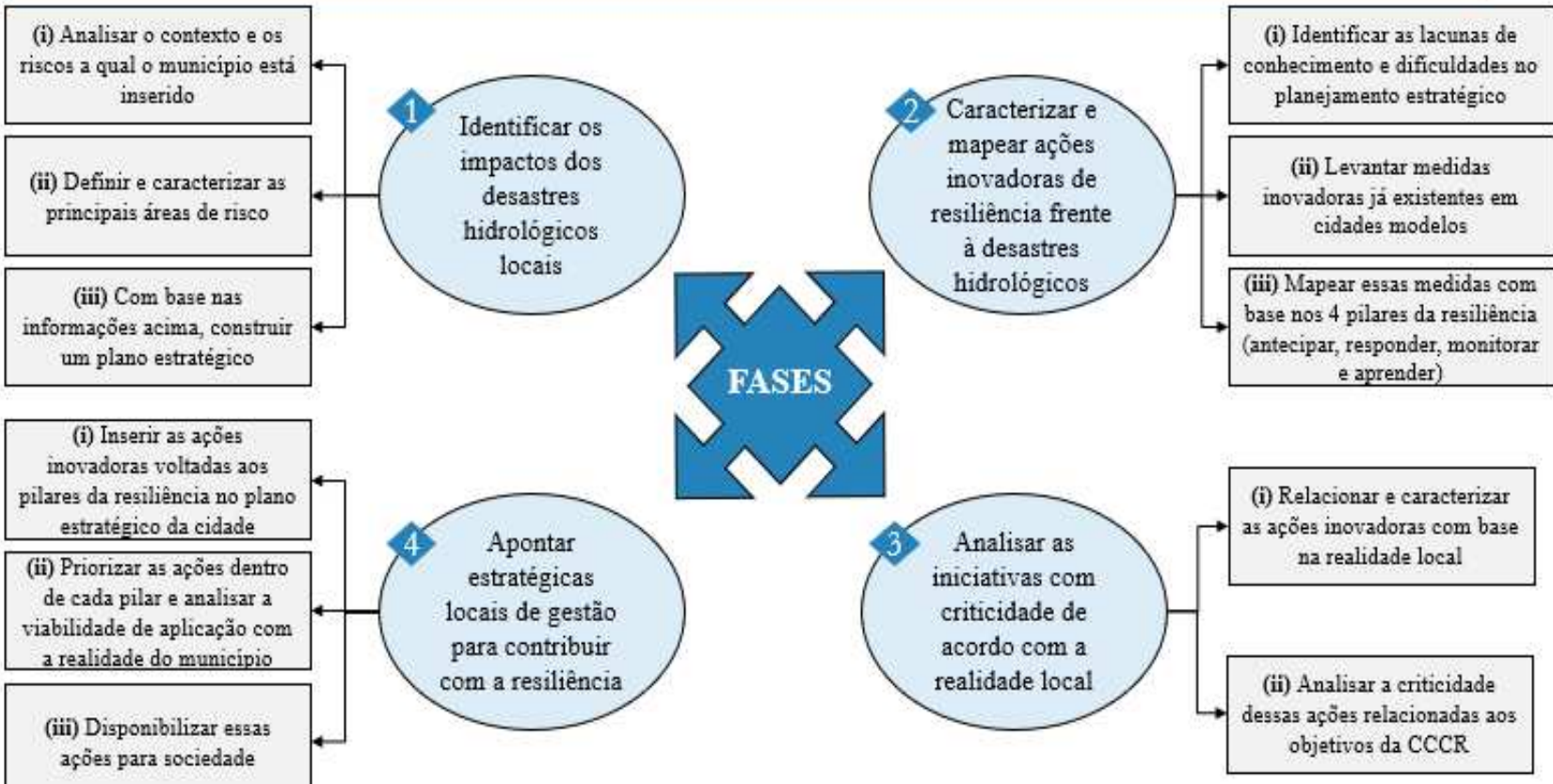

Figura 5 - Modelo de ações inovadoras para o desenvolvimento da resiliência a eventos hidrológicos nas cidades paranaenses.

No entanto, é necessário analisar criteriosamente as iniciativas inovadoras de acordo com a realidade local, pois cada cidade possui sua interpretação das capacidades de resiliências. A criticidade é utilizada para antecipar os possíveis riscos às cidades causadas pelos desastres hidrológicos, consequentemente nas capacidades de responder, monitorar e aprender com esses riscos.

Assim, para a fase 4, deverá ser realizado o alinhamento das práticas inovadoras dentro do plano estratégico das cidades, bem como a priorização e viabilidade das ações à realidade local, além de registrar e compartilhar as lições aprendidas com a sociedade. Dessa forma, por meio da proposta apresentada neste modelo de ações inovadores, é possível mapear, analisar e tornar visíveis as ações inovadoras baseadas nas experiências ao lidar com as adaptações, falhas e riscos inerentes à gestão de desastres hidrológicos, tornando as cidades mais resilientes. A validação da proposta (Figura 5) frente as partes interessadas ficarão como recomendação para pesquisas futuras.

Revista Gestão e Sustentabilidade Ambiental., v. 11, n. esp, p. 104-123, jan. 2022.

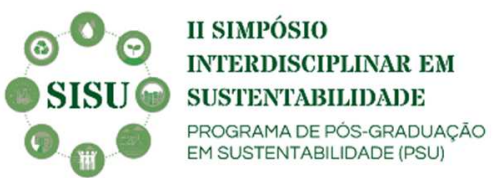




\section{CONCLUSÃO}

Esta pesquisa teve intuito de construir uma revisão sistemática da literatura no contexto de gestão de desastres hidrológicos, cidades resilientes e inovação, analisando a abordagem realizada pelo Estado do Paraná, e também, de propor um modelo de ações inovadoras utilizando a metodologia DMAIC para contribuir, desenvolver e melhorar a resiliência nas cidades paranaenses frente a desastres hidrológicos.

A revisão sistemática possibilitou identificar que há ainda poucos trabalhos que contemplam em conjunto as áreas acerca do tema aclarado no presente trabalho, havendo lacunas de conhecimento voltados ao estudo em questão. No entanto, a análise dos artigos selecionados serviu de base para o desenvolvimento do segundo objetivo deste projeto.

A utilização da metodologia DMAIC possibilitou o desenvolvimento da construção do modelo, que em uma sequência lógica levou ao resultado pretendido por meio de um objetivo principal, quatro fases e etapas descritas que auxiliarão na análise e busca por ações inovadoras de resiliência para as cidades paranaenses. Os elementos teóricos utilizados para a elaboração do modelo foram fundamentados no retrato atual das organizações paranaenses bem como suas ações de implementação da resiliência nas cidades. Esse retrato atual foi importante, pois permitiu identificar os principais recursos e atividades a serem analisadas na proposição do modelo. A validação do modelo proposto segue como recomendação para trabalhos futuros por meio da utilização de dados primários como entrevistas semiestruturadas com representantes da área.

Por fim, espera-se que o modelo proposto atinja a integração da gestão de riscos de desastres hidrológicos em diferentes setores e esferas públicas, colaborando para a prática e monitoramento da resiliência, sob uma visão clara e estratégica de longo prazo. Além de proporcionar uma gestão mais aberta e participativa com a inclusão de todas as partes interessadas, sob o objetivo de manter as funções da cidade e melhorar a resposta destes desastres a choques futuros.

\section{Agradecimentos}

Agradeço a minha orientadora, a Professora Dr. Daiane Maria De Genaro Chiroli, a Universidade Tecnológica Federal do Paraná e ao CNPq, pelo Programa Institucional de Bolsas de Iniciação Científica (PIBIC) que apoiaram e contribuíram para a construção desse trabalho. 


\section{Contribuições dos autores}

Todos os autores contribuíram de forma igualitária para o desenvolvimento desta pesquisa.

Declaração de conflito de interesses

Não há conflito de interesses.

\section{REFERÊNCIAS}

Ahern, J.; Cilliers, S.; Niemela, J. (2014). The concept of ecosystem services in adaptive urban planning and design: A framework for supporting innovation. Elsevier. Usa. Landscape And Urban Planning, 254-259.

Alem, D.; Clark, A.; Moreno, A. (2016). Stochastic network models for logistics planning in disaster relif. Elsevier, Sorocaba. European Journal Of Operational Research, 187-206.

Asad, R.; Ahmed, I.; Vaughan, J.; Von Meding, J. (2021). Traditional water knowledge: challenges and opportunities to build resilience to urban floods. International Journal Of Disaster Resilience In The Built Environment, [S.L.]. Emerald. 1-14. http://doi.org/10.1108/ijdrbe-08-2020-0091.

Barros, M. V., Ferreira, M. B., do Prado, G. F., Piekarski, C. M., \& Picinin, C. T. (2020). The interaction between knowledge management and technology transfer: a current literature review between 2013 and 2018. Journal of Technology Transfer, 45(5).

Béné, C., Wood, R. G., Newsham, A., \& Davies, M. (2012). Resilience: new utopia or new tyranny? Reflection about the potentials and limits of the concept of resilience in relation to vulnerability reduction programmes. IDS Working Papers, 2012(405), 1-61. http://doi.org/10.1111/j.20400209.2012.00405.x.

BRASIL. (2012). Política Nacional de Proteção e Defesa Civil - PNPDEC. Ministério da Integração Nacional.

Centro Universitário de Estudos e Pesquisas sobre desastres da Universidade Federal de Santa Catarina, (2012). Gestão de riscos de desastres. Florianópolis: CEPED/UFSC. 14 p.

CEPED/PR. (2018). Centro Universitário de Estudos e Pesquisas sobre Desastres. Capacity building to make cities more resilient - Class I. Portal of Distance Learning University Center for Studies and Research on Disasters. Curitiba.

CreswelL, J. W. (2010). Projeto de pesquisa: métodos qualitativo, quantitativo e misto. $3^{\text {a }}$ EDIÇÃO, Porto Alegre: Artmed, 296p.

Desouza, K. C., Flanery, T. H. (2013). Designing, planning, and managing resilient cities: A conceptual framework. Cities, 35, 89-99. http://doi:10.1016/j.cities.2013.06.003

Díaz-Delgado, C.; Iniestra, J. G. (2014). Flood risk assessment in humanitarian Logistics Process Design. Elsevier. Toluca. Journal Of Applied Research and Technology, 976-984.

Folke, C., Carpenter, S., Elmqvist, T., Gunderson, L., Holling, C. S., \& Walker, B. (2002). Resilience and sustainable development: building adaptive capacity in a world of transformations. AMBIO: $A$ journal of the human environment, 31(5), 437-440. 
Gault, F. (2018). Defining and measuring innovation in all sectors of the economy. Research policy; $47,617-22$.

Ghosh, S., Maiti, J. (2012). Data mining driven DMAIC framework for improving foundry quality a case study. Production Planning \& Control, 25(6), 478-493. https://doi:10.1080/09537287.2012.709642.

Gonçalves, M.; Fernandes, M. (2016). Risco de alagamentos no Estado do Paraná: análise da periculosidade e da vulnerabilidade. http://journals.openedition.org/confins/10974 (acesso em 14 de janeiro de 2021).

Hofmann, S. Z. (2021). 100 Resilient Cities program and the role of the Sendai framework and disaster risk reduction for resilient cities. Progress In Disaster Science, [S.L.], v. 11. Elsevier BV. http://doi.org/10.1016/j.pdisas.2021.100189. https://bi.redeexecutiva.pr.gov.br/qlikview/index.htm (acesso em 20 de março de 2021).

Hu H., Lei T., Hu J., Zhang S., Kavan P. (2018). Disaster-mitigating and general innovative responses to climate disasters: evidence from modern and historical China. International Journal of Disaster Risk Reduction; 28, 664-73.

Izumi, T.; SHAW, R.; Djalante, R.; Ishiwatari, M.; Komino, T. (2019). Disaster risk reduction and innovations. Progress In Disaster Science, [S.L.], v.2. Elsevier BV. http://doi.org/10.1016/j.pdisas.2019.100033.

Jameson, S.; Baud, I., (2016). "Varieties of knowledge for assembling an urban flood management governance configuration in Chennai, India", Habitat International, Vol. 54, 112-123.

Kahn, K. B., 2018. Understanding innovation. Business Horizon; 61,453-60.

Kobiyama, M.; Mendonça, M.; Moreno, D.A.; Marcelino, I.P.V.O; Marcelino, E.V.; Gonçalves, E.F.; Brazetti, L.L.P.; Goerl, R.F.; Molleri, G.S.F.; Rudorff, F.M. (2006). Prevenção de Desastres Naturais: Conceitos Básicos. Curitiba: Ed. Organic Trading. 109 p.

Liao, K.H., Le, T.A.;Van Nguyen, K. (2016). "Urban design principles for flood resilience: learning from the ecological wisdom of living with floods in the Vietnamese Mekong Delta", Landscape and Urban Planning, Vol. 155, 69-78.

Lima, L. L. H. R. (2020). Resiliência ao Risco. Coimbra: Estudos Cindínicos, 2020. 37 p. III Simpósio Ibero-Afro-Americano de Riscos. https://doi.org/10.34037/978-989-54942-7-9_8.

Meerow, S.; Newell, J.; Stults, M. (2015). Defining urban resilience: A review. Elsevier. Michigan (Usa). Landscape and Urban Planning, 38-49.

Miao, Q.; Popp, D. (2014). Necessity as the mother of invention: Innovative responses to natural disasters. Elsevier. USA. Journal Of Environmental Economics and Management, 280-295.

Neubauer, B. M.; Pedro Filho, F. S.; Almeida, F. M. (2019). Gestão de Serviços Públicos pelo Método DMAIC: em face da teoria u. Interscienceplace: International Scientific Journal, N/A, v. 14, n. 4, 216240.

Rodríguez-Espíndola, O.; Albores, P.; Brewster, C. (2018). Disaster preparedness in humanitarian logistics: A collaborative approach for resource management in floods. Elsevier. European Journal Of Operational Research, 978-993. 


\section{১unisul sim}

Santos, F. T. (2009). Territórios resilientes enquanto orientação de planejamento. Direção de Prospectiva e Planeamento, Lisboa.

Sokovic, M., Pavletic, D., Kern Pipan, K. (2010). Quality Improvement Methodologies - PDCA Cycle, RADAR Matrix, DMAIC and DFSS, Journal of Achievements in Materials and Manufacturing Engineering 43/1. 476-483.

Spirn, A. W. (2014). Ecological Urbanism: A Framework for the Design of Resilient Cities (2014). The Ecological Design and Planning Reader, 557-571. http://doi:10.5822/978-1-61091-491-8_50

Treichler, D.H. (2005). The Six Sigma path to leadership. New Delhi: Pearson Education.

UNISDR - International Strategy for Disaster Reduction. (2009). Terminology on Disaster Risk Reduction. http://www.unisdr.org (acesso em abril de 2021).

UNISDR. (2012). Como Construir Cidades Mais Resilientes - Um Guia para Gestores Públicos Locais. Tradução de: How to Make Cities More Resilient - A Handbook for Mayors and Local Government Leaders. Genebra, Suíça: Escritório das Nações Unidas para Redução de Riscos de Desastres, $102 \mathrm{p}$.

UNISDR. (2015). Sendai Framework for Disaster Risk Reduction 2015-2030. UNSIDR: Sendai.

UNISDR. (2017). United Nations Office for Disaster Risk Reduction. Disaster Resilience Scorecard for Cities: Detailed Level Assessment. United Nations. 118 p.

Walker, B.; Salt, D. (2012). Resilience thinking: sustaining ecosystems and people in a changing world. Washington, DC: Island Press.

Werkema, C. (2012). Criando a Cultura Lean Seis Sigma. 3 ed. Rio de Janeiro: Elsevier, 2012.

Young, C.E.F.; Aguiar, C.; Possas, E. (2014). Perdas Econômicas dos Desastres Climáticos no Estado do Rio de Janeiro, 2001-2010. Cadernos do Desenvolvimento Fluminense 5, 19-30. 